\title{
iHeartRadio's Marketing and Communication Strategies Under Covid-19
}

\section{A Bankrupted Media Company's Reaction to Unexpected Pandemic}

\author{
Yixiao Huang ${ }^{1, a, \dagger}$, Andy Kaiyuan $\mathrm{Shi}^{2, b, \dagger}{ }^{2}$, Xinyu Yuan ${ }^{2, c, \uparrow}$ \\ ${ }^{I}$ International Department, The Affiliated High School of SCNU, Guangzhou, Guangdong Province, China. \\ ${ }^{2}$ Shanghai SMIC Private School, Shanghai, China.

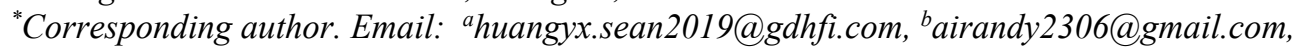 \\ cchelseayuan16@hotmail.com. \\ These authors contributed equally.
}

\begin{abstract}
For iHeartRadio, from a worldwide international online streaming service to a bankrupted radio station and bouncing back as one of the most extensive music streaming services in America, marketing strategies surely are extremely crucial to maintain their competitiveness in the market. Under the circumstance of the coronavirus outbreak, iHeartRadio, as the dominating corporation in the industry, rapidly responded to the situation by adjusting the current strategies and releasing more adaptable coping solutions. In this essay, we discuss the various strategic approaches the company employs to combat the negative effects the coronavirus pandemic could bring about. One major issue originates from the cancellation of the annual iHeartRadio music festival and live concert due to the banning of offline events during the pandemic. The company chose to display an online concert instead with artists performing at their homes for the iHeartRadio music awards, and fans were even able to interact with their idols through virtual displays. Numerous viewers were attracted for the ingenious online performance strategy, and thanks to the celebrity effect, the audience's active engagement made it a huge success. Meanwhile, the launching of the iHeartRadio app for the Apple Watch grants users more freedom and convenience to use the app in a more flexible device without carrying their phones around, encouraging people to avoid a sedentary lifestyle during the pandemic. Those strategies combined to contribute to the continuous development of iHeartRadio's stable operation during this special circumstance and spark more possible ideas for long-term benefits.
\end{abstract}

Keywords: marketing strategy, streaming service, iHeartRadio, celebrity effect

\section{INTRODUCTION}

\subsection{Background}

From an Apple-only streaming service to an all-inone iHeartRadio station, it took three years for the music streaming service to achieve this reformation. Just a short year after creating the iHeartMedia and the Clear Channel Communications by Lowry Mays and Billy McCombs, the iHeartRadio was established to become one of America's most extensive online music streaming services [1].

When the iHeartRadio was first launched on October $7^{\text {th }}, 2008$, it was only available for iPhones and iPods through the App store. A year later, the market was expanded into the Blackberry and Android system. Until
2011, the iHeartRadio successfully launched its all-inone radio, which features more than a thousand custom artist stations and live radio stations [2]. The app was then simultaneously launched on both the Xbox 360 and webOS and later the iPad. Then, the radio launched their online audio news, weather, and traffic stream in a total of 15 metropolitan cities like Los Angles, New York City, Chicago, and San Diego, branded as the 24/7 news.

In 2013, as the radio system has already gained many audiences, users, and subscribers through their years of development, they began to broaden their market internationally. Within July, iHeartRadio concurrently added stations in the United Arab Emirates like the Virgin Radio Dubai and the CHUM-FM and CFBT-FM in Canada, while also entering the Oceania market 
iHeartRadio Australia and New Zealand. It was also launched in Mexico half a decade later in 2018, covering all of North America.

After their success in the international market, they began to expand horizontally, entering different media fields. They first created the iHeartRadio Talk, featuring celebrities like Ryan Seacrest, in 2013, where users could upload their content through the Speaker. The names vary throughout the years, but the idea of a "talk radio" remained unchanged. In 2015, the iHeartRadio Family was created, which targets the younger age groups and the family as a unit. A year later, iHeartRadio plus was announced, making the streaming service on the same level as Spotify. By 2019, the app has already entered more than 250 devices and platforms available for download [3]. In the same year, the music streaming service has close to twenty-six million users in the U.S. just in September, ranking only after Apple Music, Spotify, and Pandora Radio in all of America. Now, iHeartRadio still obtains one of the world's most credible and valued ranking systems for artists worldwide and being the leading podcast publisher in the U.S., carrying more than 250,000 podcasts.

\subsection{Literature Review}

Segmanta did deep research on maximizing the value of the user feedback for iHeartRadio across multiple departments [4].

A multi-platform category voting experience was created and a UGC Meme Generator and Upic selfie generator to amplify fan participation and create social media buzz that goes with the awards [5].

TribalScale analyzed the possibility or the ideas that are given to iHeartRadio since they are coming up with ideas of developing a family-oriented app to best reach and connect with the new generation [6].

BMW partnered with iHeartMedia to find out the engagement rate of users of iHeartRadio on standard mobile display ads [7].

iHeartRadio and Macy's have been working together to find emerging music stars. They create a social voting site to vote on the nominees' fashion and give the voters the ability to shop them on Macy's [8].

iHeartRadio Australia's August 2019 campaign shows how legacy media can utilize multiple channels and human interaction to create brilliant experiences for their consumers, and they did see a boost in users [9].

iHeartRadio started a pool party inviting different stars to perform to increase their popularity and see how many users they can gain from hosting this activity [10].

Some studies aim to see whether the creation of the iHeartRadio theater in Los Angles is worthy and to test out if it can help attract more users [11].
Some article illustrates and lists the exclusive secrets behind the scenes for the living room concert that iHeartRadio presents [12].

The iHeartRadio official website depicts the contribution of iHeartRadio Music Festival including the funds it raised for charity [13].

\subsection{Research Gap}

The existed articles and papers that focus on iHeartRadio's present a common pattern of focusing on the operating strategies of this company, such as the communication strategies study, the users' features study, and the research of the way iHeartRadio gets profit. Besides these documents about how a media company reaches its consumers, the field of iHeartRadio's operating strategies under the current circumstance of covid-19 remains untouched. The topic of this paper iHeartRadio's communication strategies in North America under the influence of covid-19 - will fill the black and somehow provide some feasible ideas for media companies like iHeartRadio to deal with the pandemic.

\subsection{Importance of the Research}

The pandemic surely has a destructive and continuous impact worldwide in numerous aspects, especially in North America, leaving many streaming media corporations facing a drastic decline in revenue making, including the iHeartRadio. Thereby, increasing profits in the short term appears to be more urgent than ever. To help cope with this problem of iHeartRadio, analyzing the advantages and drawbacks of the current strategies is imperative since we will figure out ways to improve only when we get a full picture of the situation and have a sophisticated understanding of it.

Moreover, as a dominating company in the media and radio industry, the case of iHeartRadio would spark new ideas and inspirations for fellow companies in terms of adjusting marketing and communication strategies under this special circumstance. This study, therefore, could serve to revitalize the industry and exert a far-reaching influence in the long run.

\subsection{Research Subject \& Methodology}

Given the case of iHeartRadio, we explore its communication strategy in North America under the outbreak of coronavirus and analyze the favored and repelling aspects accordingly to contribute to further development of the company and evoking ideas for other companies in the media industry.

This study adopts the quantitative research method of case analysis. The researchers can only study the benefits and drawbacks of communication strategies based on 
observation without accurate data due to the protection of confidential information.

\section{DISCUSSION}

\subsection{Online Concerts}

As a result of covid-19, several popular concerts and music festivals held by iHeartRadio were delayed or canceled in 2020. To minimize the impact that the pandemic circumstance can bring to the company, iHeartMedia gathered music performances and turned them into online events as replacements for offline concerts. The company held the iHeart Living Room Concert and the 2020 iHeartRadio Music Festival to fill the blank of concerts under covid-19 and simultaneously contribute to the control of the epidemic by keeping the audience safe at home. This act ensured an enjoyable experience for the audience base, ultimately collecting the budget for the charities to fight the disease.

Taking the iHeartRadio Music Awards, which was canceled due to the pandemic, The iHeart Living Room Concert was held on March 29th, 2020. The English songwriter Elton John collaborated with Fox to present this music festival and broadcasted it on the iHeartRadio app. Celebrities like Sam Smith, Shawn Mendes, Billie Eilish, and Ellen DeGeneres were invited to show up at the concert, performing at different places on the screen. Famous band the Backstreet Boys, also reunited and sang their signature song "I Want It That Way", pushing the show to a climax [12]. With all these stars performing at their homes, the relaxing atmosphere created a cozy mood for the people who watched and attended this show, giving them relief from the current intense global situation. According to the data collected by the producers, the numerous viewers attracted to this show and the donation system raised more than $\$ 10$ million for the charities [13]. The success of this action maintained the interaction between artists and fans and supported the worldwide fight against covid-19.

As a tradition of iHeartRadio, the iHeartRadio Music Festival welcomed its 10th anniversary in 2020, and the event was held online. Unlike the living room concert, this time, the artists were asked to perform on the stages in Los Angeles, and Nashville, where there were screens consist of thousands of virtual audiences, and their performance was filmed and played on the app. International stars like BTS, Migos, and Miley Cyrus came to the festival and provided exceptional listening experiences for audiences across the country. A notable detail of this event is that the fans watching the show could be selected and get the chance to appear on the virtual fan wall and be serenaded by their idols [14]. The interesting designs of this music festival effectively provoked people's excitement toward musical performances and kept the company operating actively despite the influence of covid-19.

\subsection{Becoming an Apple Watch Application}

In terms of ways of transmission, iHeartRadio officially launched for Apple Watch in 2020, meaning that listeners could get access to their favorite music and other content anytime and anywhere [15]. With as many as over 95 million registered users, iHeartRadio needed improving users' quality and efficiency of music listening. The mass consumer base compels the company to develop the app in more flexible ways to make them stick to iHeartRadio's products and services in the long run.

The user experience was guaranteed since listeners could access the app's features include Search, Your Library, Recently Played, etc. The adequate capacities on the watch assured that users were allowed to enjoy roughly the same functions of the iOS app. A notable feature of this new transmission policy is that the app could work on its own given the absence of your iPhone. In other words, it is no longer just an iOS companion app but an app that can function independently with no strings attached. This alternative gives listeners the freedom to leave their phones behind and still listen to their favorite music and podcasts [15]. It is a lot of help for those who do not prefer to carry their phones around all day long, promoting a healthier way of living. We all know that iWatch automatically records your exercise index, which could account for why many people wear Apple Watch while working out. Adding on to this function, the availability of iHeartRadio on the watch will encourage users to listen to music while doing exercise; the combination will provide psychological comfort for intense exercise, and as a result, amplifying the effect as well as changing the sedentary lifestyle by spurring them to work out.

This new transmission approach enables users to access the app more conveniently by combining music to a frequently used device. In this way, if you are in rush hours, in the middle of jogging, or any moment where your hands are tight but eager to listen to music, you can save the effort of pulling your phone out of the bag and listen to the music directly by operating of the watch. Therefore, the users would gradually rely on the app more often as one can use it without considering whether it is the appropriate time or place. Moreover, making the app available to a more portable device could appeal to a wider range of consumers for the iHeartRadio. It opens up more possibilities since the Apple users who previously haven't downloaded the iOS app might find this app handy on the watch and eventually become an actual app register. The Apple company could also benefit from this collaboration. By exploiting more functions on the watch, the company will push people to become more attached to Apple Watch due to its increasing usefulness. 


\subsection{Celebrity Effect}

The flexible adaptations, especially online events, made by iHeartMedia to mitigate the damage of the pandemic successfully boosted the company's economic condition and overall development. From the perspective between artists and fans, they helped the artists keep gaining the public attention and gave the fans the chance to watch their favorite celebrities perform with the absence of tangible offline shows. As for society, the funds raised by the performances served a key role in combating the spread of coronavirus. Being the host, iHeartRadio also received significant implicit profit from the online events, as they kept on leaving a good reputation for its users and potential subscribers like the audiences, making progress on its road of return from the bankruptcy. Besides, the designers can make a few changes to the programs to improve the watching experience if more online shows will be needed shortly. The rewards for the fans like those in the music festival 2020 can be applied to other shows since the stimulating scenes like the direct interactions between fans and idols will impact the popularity of any show positively. For the next online show, the company should consider adding the part that allows the audience to be randomly selected to talk to the artists face-to-face in front of the screen. To achieve a more vivid watching experience, it is also a choice to present the shows with virtual reality and provide the viewers with relatively cost-effective devices to access the performances.

As the unexpected pandemic surprised the whole world at the beginning of 2020, billions of people's lives were tremendously affected. Traveling restrictions were set, public gatherings were banned, nations were locked down, everyone was panicking. The pandemic spread its influence on millions of families and countess publics figures were forced to stop all actions. The four major professional sports leagues in the United States and Canada were forced to suspend their seasons, tv shows and movies in the making progress were postponed, live tours and concerts by musicians were canceled. To minimize the loss, many media companies like iHeartRadio came out to support the government, protect the citizens, and make a profit at the same time. At the Nashville and Los Angles concert, artists performed in front of virtue audiences. They used this opportunity to adhere to social guidelines that benefit the social wellbeing of citizens and help the control of the pandemic at that time. With the words coming out of the mouth of idols like BTS, Kane Brown, and Miley Cyrus, it sometimes works even better than government officials' guidelines. According to iHeartMedia's president of entertainment enterprises John Sykes in an extensive interview with Variety, the Livingroom Concert will be "completely scrubbed and cleaned with no audience." By doing this, the company can spread its names worldwide and favor the government in helping to promote healthy guidelines to keep citizens safe in a crisis like this. Like the entertainment editor, P. Claire Dodson, said: "We figured out a strategy to put safety first and still deliver a live music experience." IHeartRadio successfully found a way to spread and undergo safety protocols, make a better reputation, and deliver music simultaneously by using these online concerts to limit face-to-face contacts by artists and audiences while still communicating through the music and the ideas.

\section{CONCLUSION}

The information above is concluded from the example of iHeartRadio to give an analysis of the ongoing marketing strategies and some suggested ideas that might stimulate the company's development under the pandemic situation, intending to be referable for iHeartMedia as well as other media firms. Dealing with the absence of offline events, making adjustments to the communication strategies, and focusing on the online market is the most direct way that can be applied. As a replacement for the conventional iHeartRadio Music Awards, the iHeart Living Room Concert attracted the audiences differently than all the performers were in their "casual mode", singing at their home, with video streaming that displays their voice. Besides, the online version of the iHeartRadio Music Festival gathered celebrities worldwide to interact with fans, and the program also raised over 10 million dollars for combating the epidemic. After holding two online events in a row, iHeartRadio retained the public's attention in a way that supports the global fight against covid-19. All these methods adopted by iHeartMedia can be attributed to the celebrity effect, which guaranteed the influence that these events have. By inviting international superstars to perform and advocate under the name of iHeartRadio, the company's voice can be easily heard by more people and therefore promoted the prevalence of their products. Similarly, the claims made by the stars about how to react to the pandemic can be even more persuasive than government official documents sometimes, so the safety awareness of the public can be improved in this way under this dangerous period. Another approachable method of broadening the market of this application used by iHeartMedia is to adapt it to Apple Watch. The convenient user experience of the Apple Watch enables iHeartRadio to occupy much more time in people's daily lives. Combining with the personalized function of the watch, the quality of this app is significantly increased. Consequently, the result will be the growing popularity of the app. As time goes on, all these actions will end up letting iHeartRadio assimilate into much more people's lives, regardless of the negative impact of covid-19.

\section{REFERENCES}

[1] E, Griffith, Bob Pittman doesn't believe streaming will kill radio. But he's built a massive streaming 
service, just in case, Fortune, 2014. DOI: https://fortune.com/2014/06/17/iheartradio-clearchannel-bobpittman/\#: :text=Bob\%20Pittman\%20doesn't\%20b elieve,streaming $\% 20$ service $\% 2 \mathrm{C} \% 20$ just $\% 20 \mathrm{in} \% 2$ 0case\&text $=$ And\%20then\%20there's\%20iHeartRad io $\% 2 \mathrm{C} \% 20$ the,crossed $\% 2050 \% 20$ million $\% 20$ regist ered\%20users

[2] B. Sisario, Clear Channel Renames Itself iHeartMedia in Nod to Digital, The New York Times, 2014.2 DOI: https://www.nytimes.com/2014/09/17/business/me dia/embracing-digital-brand-clear-channelrenames-itself-iheartmedia.html

[3] L. E. Jasinki, J. M. Linsday, iHeartMedia, Inc., Texas State Historical Association DOI:https://www.tshaonline.org/handbook/entries/i heartmedia-inc

[4] iHeartRadio, Segmanta. DOI: https://segmanta.com/casestudies/iheartradio/

[5] iHeartRadio, Segmanta. DOI: https://segmanta.com/casestudies/iheartradio/

[6] iHeartRadio Family Case Study, TribalScale. DOI: https://ts-website-assets.s3.amazonaws.com/casestudies/TribalScale-iHeartRadio-CaseStudy.pdf

[7] BMW Case Study, AdsWizz., 20 May 2020, DOI: https://www.adswizz.com/bmw-case-study/

[8] Macy's iHeartRadio Rising Star Contest (Case Study), Zoomph, 7 November 2018. DOI: https://zoomph.com/blog/macys-iheartradio-risingstar-case-study/

[9] iHeartRadio Australia Boosted Monthly Active Users by $17 \%$, Braze. DOI:

https://www.braze.com/customers/iheartradio-casestudy

[10] The iHeartRadio Ultimate Pool Party Presented by Visit Florida Returns to Fontainebleau's Bleaulive for Year Two, iHeartMedia, Inc. DOI: https://www.iheartmedia.com/press/iheartradioultimate-pool-party-presented-visit-florida-returnsfontainebleaus-bleaulive-year

[11] A. Ashford, iHeartRadio Theater Los Angeles "Hearts" DiGiCo, rAVe [PUBS], 19 October 2020. DOI: https://www.ravepubs.com/iheartradiotheater-los-angeles-hearts-digico/

[12] M. Schneider, Fox, IHeart Execs Reveal Secrets Behind 'Living Room Concert' Performances (EXCLUSIVE), Variety, 9 April, 2020. DOI: variety.com $/ 2020 / \mathrm{tv} /$ news/iheart-living-room- concert-fox-behind-the-scenes-mariah-carey1203549088/.

[13] H. Brooks, Elton John-Hosted IHeart Living Room Concert For America Raises \$10 Million, IHeartRadio, 1 April, 2020. DOI: news.iheart.com/featured/living-room concert/content/2020-04-01-elton-john-hostediheart-living-room-concert-for-america-raises-8million/.

[14] T. Fields, 2020 IHeartRadio Music Festival Lineup Revealed, IHeartRadio, 21 July 2020. DOI: www.iheart.com/content/2020-07-21-2020iheartradio-music-festival-lineuprevealed/\#: : :text=The $\% 202020 \% 20 \mathrm{iHeartRadio} \%$ 20Music\%20Festival,all\%20hosted\%20by\%20Rya n\%20Seacrest.

[15] T. Fields, iHeartRadio App Launches For Apple Watch, Media in Canada, 28 September, 2020. DOI: https://www.iheart.com/content/2020-09-28iheartradio-app-launches-for-apple-watch/ 